\title{
Hospitalization rates for human metapneumovirus infection among 0- to 3-year-olds in Gipuzkoa (Basque Country), Spain
}

\author{
G. CILLA ${ }^{1 *}$, E. ONATE ${ }^{2}$, E. G. PEREZ-YARZA ${ }^{2}$, M. MONTES ${ }^{1,3}$, D. VICENTE ${ }^{1,3}$ \\ AND E. PEREZ-TRALLERO ${ }^{1,3,4}$ \\ ${ }^{1}$ Servicio de Microbiología, Hospital Donostia, San Sebastián, Spain \\ ${ }^{2}$ Departamento de Pediatría, Hospital Donostia, San Sebastián, Spain \\ ${ }^{3}$ CIBER Enfermedades Respiratorias CB 06/06-26, San Sebastián, Spain \\ ${ }^{4}$ Departamento de Medicina Preventiva y Salud Pública, Facultad de Medicina, Universidad del País Vasco, \\ San Sebastián, Spain
}

(Accepted 25 March 2008; first published online 18 April 2008)

\section{SUMMARY}

Numerous studies have been published on human metapneumovirus (HMPV) infection, but few have been population based. The main aim of this study was to estimate the incidence rate of hospitalization for community-acquired HMPV infection in infants and children aged $<3$ years. Between July 2004 and June 2007, 796 episodes (742 patients) of community-acquired acute respiratory infection were hospitalized. HMPV was detected in 90 episodes $(11 \cdot 3 \%)$. Fifty-nine episodes occurred in infants aged $<1$ year. The mean length of hospital stay was $6 \cdot 2$ days (range 2-31 days). Thirteen children required admission to the intensive care unit. Viral co-infections were detected in 46 episodes $(51 \cdot 1 \%)$. The incidence rate of hospitalization per 1000 inhabitants was $2 \cdot 6(95 \%$ CI $2 \cdot 1-3 \cdot 2)$, lower than that for respiratory syncytial virus, but higher than that observed for the influenza and parainfluenza viruses. HMPV is a major respiratory pathogen that leads to a high hospitalization rate.

\section{INTRODUCTION}

Human metapneumovirus (HMPV) is a novel paramyxovirus discovered in 2001 and closely related to avian pneumovirus [1,2]. Accumulating evidence has shown that HMPV has a worldwide distribution and seasonal circulation, that it is a common respiratory pathogen in childhood, and that it contributes substantially to the aetiology and pathogenesis of lower respiratory tract infection (LRTI) [2-7]. Numerous studies have focused on the clinical and epidemiological characteristics of HMPV infection, but few have been population based, and consequently the

\footnotetext{
* Author for correspondence: Dr G. Cilla, Servicio de Microbiología, Hospital Donostia, Paseo Dr. Beguiristain s/n, 20014 San Sebastián, Spain.

(Email: carlosgustavo.cillaeguiluz@osakidetza.net)
}

available information on the incidence and burden of this disease remains scarce. In addition, few studies have been conducted over several years, which is essential to adequately evaluate incidence rates, given that the intensity of HMPV circulation may vary considerably in distinct seasons [8-10]. The main aim of this study was to accurately estimate the incidence rates of hospitalization for community-acquired HMPV infection in an unselected and well-defined population of infants and children aged $<3$ years from an area of northern Spain.

\section{METHODS}

\section{Patients}

This prospective study was performed in the regions of San Sebastián, Tolosa and Urola-Costa (province 
of Gipuzkoa, Autonomous Community of the Basque country, northern Spain). The infants and children from these regions provided population-based data (population 405745 inhabitants, of which 11696 were aged $<3$ years old). Hospital Donostia (San Sebastián, 79 paediatric beds), is the only public hospital in this area and attends about $97 \%$ of paediatric hospitalizations. This study was performed between July 2004 and June 2007. Candidates for inclusion in this study were those aged 0-3 years attending the hospital's Paediatric Emergency Department for acute respiratory infection (ARI). The study period was divided into three seasons: July 2004-June 2005, July 2005-June 2006, and July 2006-June 2007.

During admission to the Paediatric Emergency Department a nasopharyngeal aspirate (NPA) was obtained from all infants and children hospitalized for ARI, both because these patients formed part of this prospective study of respiratory virus infection and because it is the hospital's policy to detect patients infected with respiratory syncytial virus (RSV) to allow for cohorting. Patients who lived outside the study health district, and those from whom an NPA was obtained after $72 \mathrm{~h}$ of hospitalization (probable nosocomial infection) were excluded. Samples obtained from the same patient at an interval of $<8$ weeks were considered to be within the same episode unless the medical records showed successive ARI and virological study showed infection by a new virus. This study was approved by the Ethics Committee for Clinical Research of Hospital Donostia.

\section{Virological studies}

The samples were immediately sent to the laboratory and were divided in two aliquots, one of which was stored at $2-8{ }^{\circ} \mathrm{C}$ and analysed for diagnostic purposes within $48 \mathrm{~h}$ of extraction, while the other was stored at $-80{ }^{\circ} \mathrm{C}$ for additional research studies. In all NPA, RSV antigen detection was performed, using a commercial immunochromatographic method (Binax, Portland, ME, USA). RSV, influenza A and B, parainfluenza viruses types 1-3 and adenovirus were investigated through culture using the rapid shell vial techniques on MDCK, A-549 and LLC-MK2 cell lines. Similarly, the presence of RSV [11], influenza A and B [11, 12], parainfluenza 1-4 [11], rhinovirus [13], respiratory coronaviruses [14], and coronavirus NL63 [15] were investigated using multiplex reverse transcriptase-polymerase chain reaction (RT-PCR) methods, the presence of adenovirus was investigated with a PCR method [16]. RT-PCR for HMPV was performed using primers derived from the $F$ gene [17]. Viral RNA was obtained with an automatic BioRobot ${ }^{\circledR}$ M48 extractor (Qiagen GmbH, Hilden, Germany) using the MagAttact ${ }^{\circledR}$ Virus Mini M48 kit. Transcription of RNA to cDNA was performed with M-MuLV reverse transcriptase (USB Corp., Cleveland, OH, USA), using random primers. Each PCR run included a negative control (water control) that was treated identically to the clinical samples throughout. All procedures were performed with the usual precautions to avoid contamination.

\section{Statistical analysis}

To calculate the incidence rate of hospitalization by age groups, data from the population living in Gipuzkoa in 2006 were used (Basque Institute of Statistics). We calculated $95 \%$ exact binomial confidence intervals. Data on the demographic characteristics of each patient (age, sex, date of admission, etc.), whether the patient was hospitalized, place of hospitalization [admission to the paediatric intensive care unit (PICU)] or another ward) and duration of hospitalization, as well as the results of virological tests, were gathered using the hospital's computerized registries. Discharge diagnoses were recorded for all patients enrolled in this study. The $\chi^{2}$ test was used to compare percentages (qualitative variables) with application of Yates' or Fisher's corrections (two-tailed) when required. A $P$ value of $<0.05$ was considered statistically significant.

\section{RESULTS}

During the study period, 796 episodes of communityacquired ARI requiring hospitalization were investigated, corresponding to 742 infants and children aged $<3$ years ( 30 patients had two episodes and 11 patients had three or more episodes). There were 449 episodes in boys and 347 in girls. Most of the episodes $(n=539,67 \cdot 7 \%)$ occurred in infants aged $<1$ year, corresponding to 386 aged $<6$ months (Table 1). A total of $60 \cdot 2 \%$ of the episodes investigated $(n=479)$ occurred between November and February. In 95 episodes, the patients were admitted to the PICU $(11.9 \%)$, while the remainder were admitted to other wards. An identifiable virus was found in 612 episodes (76.9\%), including RSV in 350, rhinovirus in 166 , HMPV in 90 (see below), parainfluenza virus in 60, influenza virus in 36, adenovirus in 29 and 
Table 1. Number of cases and mean annual incidence of hospitalization (rate per 1000 inhabitants) due to community-acquired acute respiratory infections in 0- to 3-year-olds in Gipuzkoa (Basque Country, Spain), July 2004 to June 2007

\begin{tabular}{clllll}
\hline \hline & Total hospitalized & RSV & Influenza* & Parainfluenza & HMPV \\
\hline Hospitalized infants aged & 386 & 231 & 14 & 23 & 39 \\
$<6$ months (rate) & $(65 \cdot 9 / 1000)$ & $(39 \cdot 4 / 1000)$ & $(2 \cdot 4 / 1000)$ & $(3 \cdot 9 / 1000)$ & $(6 \cdot 7 / 1000)$ \\
Hospitalized infants aged & 153 & 63 & 8 & 18 & 20 \\
6-11 months (rate) & $(26 \cdot 1 / 1000)$ & $(10 \cdot 8 / 1000)$ & $(1 \cdot 4 / 1000)$ & $(3 \cdot 1 / 1000)$ & $(3 \cdot 4 / 1000)$ \\
Hospitalized children aged & 153 & 36 & 12 & 14 & 21 \\
12-23 months (rate) & $(12 \cdot 9 / 1000)$ & $(3 \cdot 0 / 1000)$ & $(1 \cdot 0 / 1000)$ & $(1 \cdot 2 / 1000)$ & $(1 \cdot 8 / 1000)$ \\
Hospitalized children aged & 104 & 20 & 2 & 5 & 10 \\
24-35 months (rate) & $(9 \cdot 0 / 1000)$ & $(1 \cdot 7 / 1000)$ & $(0 \cdot 2 / 1000)$ & $(0 \cdot 4 / 1000)$ & $(0 \cdot 9 / 1000)$ \\
Total no. of cases and & 796 & 350 & 36 & 60 & 90 \\
mean annual incidence & $(22 \cdot 7 / 1000)$ & $(10 \cdot 0 / 1000)$ & $(1 \cdot 0 / 1000)$ & $(1 \cdot 7 / 1000)$ & $(2 \cdot 6 / 1000)$ \\
\hline \hline
\end{tabular}

RSV, Respiratory syncytial virus; HMPV, human metapneumovirus.

* Influenza includes influenza virus A and B.

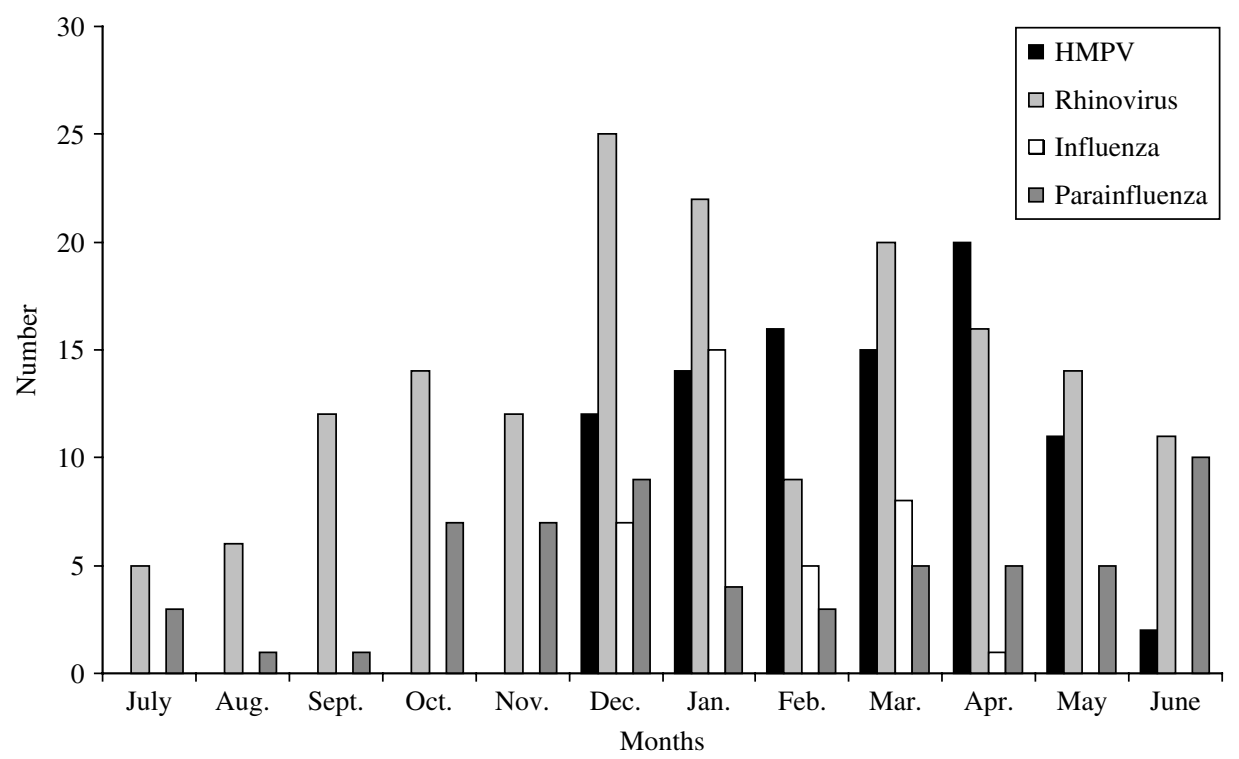

Fig. 1. Monthly distribution of the accumulated number of human metapneumovirus (HMPV)-positive episodes and other respiratory viruses in a 3-year period (July 2004 to June 2007). Respiratory syncytial virus (RSV) was not represented to avoid distorting the figures (the RSV figures were: six episodes in October, 54 in November, 172 in December, 73 in January, 25 in February, 10 in March, four in April, one in May, three in June, one in July and one in August). Note that influenza includes influenza virus $\mathrm{A}$ and $\mathrm{B}$.

coronavirus in 26. Viral co-infections were identified in 137 episodes $(17 \cdot 2 \%)$.

\section{Epidemiological characteristics of HMPV infections}

HMPV was detected in $11.3 \%$ of the episodes, the corresponding figures being $31(12.2 \%), 30(10.8 \%)$ and $29(11 \cdot 0 \%)$ in the 2004-2005, 2005-2006 and 2006-2007 seasons respectively. HMPV was detected in 50 episodes in boys $(11 \cdot 1 \%)$ and in 40 episodes in girls $(11 \cdot 5 \%)$, the mean age (s.D.) being $10 \cdot 1( \pm 8 \cdot 5)$ months. HMPV was detected in patients hospitalized between December and June, it was the virus most frequently detected in April $(42.6 \%, 20 / 47$ of the episodes studied) and the second, after rhinovirus, in May $(25.0 \%, 11 / 44)$ and March $(21.8 \%, 15 / 69)$ (Fig. 1). Two-thirds of the episodes in which HMPV was detected corresponded to infants aged $<1$ year (59/90). However, no significant differences were found in the percentage of HMPV-positive episodes 
among patients of different ages, this virus being detected in $10 \cdot 1 \%(39 / 386)$ of infants aged $<6$ months, in $13 \cdot 1 \%(20 / 153)$ of those aged $6-11$ months and in $12 \cdot 1 \%(31 / 257)$ of children aged $12-35$ months $\left(\chi^{2}\right.$, 2 D.F. $1 \cdot 18, P=$ n.s.). Co-infections of HMPV with other viruses were identified in $46(51 \cdot 1 \%)$ episodes, the most frequently associated viruses being rhinovirus $(n=23)$ and $\operatorname{RSV}(n=18)$.

\section{HMPV hospital and PICU admission}

Of the 90 episodes of hospitalization caused by HMPV, $75(83.3 \%)$ occurred in patients with one LRTI, $11(12 \cdot 2 \%)$ in patients with a febrile illness with involvement of the upper respiratory tract or undefined respiratory tract localization and four occurred in patients with acute asthma exacerbations. The mean length of hospital stay was 6.2 days (range 2-31 days), and consequently we estimated that HMPV accounted for 186 days of hospitalization per year in infants and children aged $<3$ years in our region.

HMPV was detected in $11 \cdot 7 \%(75 / 639)$ of patients hospitalized for LRTI. After excluding patients with more than one discharge diagnosis of LRTI (i.e. bronchitis and pneumonia), HMPV was detected in $10 \cdot 3 \%(38 / 370)$ of patients hospitalized for bronchiolitis, in $12 \cdot 3 \%(20 / 163)$ of those hospitalized for pneumonia and in $22.0 \%(11 / 50)$ of those hospitalized for acute bronchitis. Because of the severity of the episodes, 13 patients required admission to the PICU (14.4\% of the episodes with HMPV and 13.7\% of the 95 episodes admitted to the PICU), of which five occurred in infants aged $<6$ months. In seven of these 13 patients, viral co-infections were detected, RSV being identified in three patients, rhinovirus in three patients, and influenza A virus in one patient. One patient was hospitalized on two occasions during the course of HMPV infection (episodes of acute bronchitis at the ages of 5 and 13 months). There were no deaths.

\section{Population-based incidence of hospitalization due to HMPV infection}

The mean annual incidence rates of hospitalization due to community-acquired HMPV infection per 1000 inhabitants in the study period were 6.7 (95\% CI $4 \cdot 8-9 \cdot 1)$, 5.0 (95\% CI 3·8-6.5), $3 \cdot 4$ (95\% CI 2·7-4.2) and $2 \cdot 6(95 \%$ CI $2 \cdot 1-3 \cdot 2)$ for patients aged less than $6,12,24$ and 36 months, respectively. No major
Table 2. Incidence of hospitalization (rate per 1000 inhabitants) due to community-acquired human metapneumovirus infection in Gipuzkoa, Autonomous Community of the Basque Country, Spain, according to age and study season (July 2004-June 2007)

\begin{tabular}{clll}
\hline \hline & $\begin{array}{c}\text { July 04- } \\
\text { June 05 }\end{array}$ & $\begin{array}{l}\text { July 05- } \\
\text { June 06 }\end{array}$ & $\begin{array}{l}\text { July 06- } \\
\text { June 07 }\end{array}$ \\
\hline Hospitalized infants & 15 & 12 & 12 \\
aged <6 months (rate) & $7 \cdot 7 / 1000$ & $6 \cdot 1 / 1000$ & $6 \cdot 1 / 1000$ \\
Hospitalized infants & 6 & 8 & 6 \\
aged 6-11 months (rate) & $3 \cdot 1 / 1000$ & $4 \cdot 1 / 1000$ & $3 \cdot 1 / 1000$ \\
Hospitalized children & 6 & 6 & 9 \\
aged 12-23 months (rate) & $1 \cdot 5 / 1000$ & $1 \cdot 5 / 1000$ & $2 \cdot 3 / 1000$ \\
Hospitalized children & 4 & 4 & 2 \\
aged 24-35 months (rate) & $1 \cdot 0 / 1000$ & $1 \cdot 0 / 1000$ & $0 \cdot 5 / 1000$ \\
Hospitalized children & 31 & 30 & 29 \\
aged <3 years (rate) & $2 \cdot 7 / 1000$ & $2 \cdot 6 / 1000$ & $2 \cdot 5 / 1000$ \\
\hline \hline
\end{tabular}

variations were found in the distribution of the incidence of hospitalization per season for each group (Table 2). Comparison of the mean annual incidence of hospitalization per 1000 inhabitants obtained in the study period for HMPV, RSV, influenza A and $B$ viruses and parainfluenza viruses are shown in Table 1. During the study period, the ratio between the incidence of hospitalization per 1000 inhabitants obtained for RSV and that obtained for HMPV was $3 \cdot 8$, with a tendency to decrease with increasing age, being 5.9 times higher in infants aged $<6$ months, $3 \cdot 2$ times higher in those aged 6-11 months and 1.8 times higher in children aged 12-35 months. In all age groups considered, the mean annual incidence of hospitalization for HMPV was higher than that observed for the influenza and parainfluenza viruses.

\section{DISCUSSION}

The general characteristics of HMPV infection highlighted by this study show strong similarities with those that have emerged from broad, previously published studies. HMPV was detected in $11.3 \%$ of the episodes investigated and in $11.7 \%$ of those hospitalized for LRTI. These figures are similar to those reported in Australia [3], the United States [4], Israel [18], and Europe [19, 20]. Although HMPV was detected sporadically in the inter-epidemic period, circulation of this virus had a clear seasonal pattern, with a predominance in winter and at the beginning of spring, partially overlapping with the RSV season. The greatest number of respiratory infections due to 
HMPV were detected in the trimester March-May, HMPV RNA being found in more than a quarter of the ARI episodes investigated in this period (28.8\%). A similar seasonality has been observed in studies performed in temperate regions [10, 17, 19, 20]. An interesting finding of this study was an unusually high frequency $(51 \cdot 1 \%)$ of HMPV detection together with other respiratory viruses (viral co-infections). Some recent studies have also found a significant frequency $(>30 \%)$ of co-infections of HMPV with other respiratory viruses $[8,19,21]$. These results are at least partly due to the increasingly frequent use of molecular diagnostic methods for a greater number of viruses. The most frequently found co-infections were dual infections with HMPV and RSV or rhinovirus, probably due to the high prevalence of both viruses in the episodes investigated and to the seasonal circulation of these viruses, which is partly simultaneous. Recurrent episodes of hospitalization due to HMPV infection were found in only one patient. Reports of recurrent HMPV infections have in general been scarce $[4,10,20]$. These studies show that HMPV reinfections rarely give rise to severe illnesses, supporting the existence of some degree of cross-protective immunity between different HMPV lineages [10].

To estimate the impact of HMPV infection, the number of HMPV isolates obtained has often been compared with that of other viruses in which the burden of disease is well known, usually RSV, but very few studies have been population based. To our knowledge, the only HMPV population-based study previously conducted in Europe is a recent cohort study in children in the community [22], and no prior studies have examined the incidence of hospitalization. The stengths of the present study are that it was performed in three consecutive seasons, that it included almost all the 0- to 3-year-olds hospitalized for ARI, and that it investigated for the presence of HMPV and other viruses through molecular methods. Moreover, this study was performed in a region that provided population-based data, allowing the incidence of hospitalization attributable to HMPV to be accurately estimated.

The incidence of hospitalization for RSV and influenza virus was similar to those previously observed for these viruses in our geographical area, which were also in line with those reported in other developed countries [23, 24]. These results also support the consistency of the incidence rates obtained for HMPV. The mean annual incidence of hospitalization for HMPV in the 0-3 years age group $(2 \cdot 6 / 1000$ inhabitants) was higher than that observed in the United States $(1 \cdot 1$ and $1 \cdot 3 / 1000$ inhabitants for infants aged $<12$ months and children aged 12-23 months, respectively) [25] but was lower than that observed in studies performed in Hong Kong (4.4/ 1000 inhabitants aged $<6$ years) [6] and South Africa $(5 \cdot 7 / 1000$ human immunodeficiency virus uninfected children) [7]. Significant annual variations in the circulation rate of HMPV have been reported and are probably partially responsible for the different incidence rates reported in these studies. Indeed, of the three above-mentioned studies, two were conducted over only one season $[6,25]$. Other reasons that might help to explain these differences are differences in health-care systems and socioeconomic conditions among countries, as well as in policies of childhood hospitalization.

In agreement with the results of Mullins et al. [25], our results indicate that the incidence of hospitalization due to HMPV is clearly lower than that observed for RSV, and that overall the incidence of hospitalization for HMPV is four times lower in children aged $<3$ years. Although the mean incidence of hospitalization was greater for RSV than for HMPV in all the age groups considered, this difference was greatest in the first few months of life and rapidly decreased with age until the incidence of RSV was only 1.8 times higher than that of HMPV in children aged $12-35$ months. This decrease is coherent with the finding in numerous series that the mean age of cases of HMPV infection is higher than that of cases of RSV infection [6, 8, 18, 25]. In contrast with the incidence of RSV, that of HMPV was greater than that of other important viral respiratory pathogens such as the influenza and parainfluenza viruses in all the age groups analysed, suggesting a major role of this new respiratory virus in the pathogenesis of severe LRTI.

The present study has some limitations. First, we used frozen specimens for RT-PCR and the molecular techniques did not include detection of potential PCR inhibitors. In addition, while the PCR procedure used is an efficient method for detecting HMPV and detects viruses of both subgroups and both genotypes [17], it is based on the detection of a single gene. These factors may have diminished the yield, and consequently the results obtained should be interpreted as minimal. Second, when comparing incidence rates among different viruses, the sensitivities of detection methods for each virus may differ. Finally, another limitation is that some cases may have been the result 
of prolonged viral excretion after a previous infection. However, HMPV excretion in immunocompetent individuals seems to be short lived [22, 26], making detection unrelated to the current infection unlikely. New investigations based on quantification of viral load could provide key information on the role of this virus in the future.

In conclusion, the present study determined the mean annual incidence of hospitalization due to HMPV infection in the first 3 years of life over a prolonged period in a developed region of Europe. The impact of HMPV infection is clearly lower than that of RSV, especially in infants aged $<1$ year. However, our data indicate that in the $0-3$ years age group HMPV is a major respiratory pathogen that, in most seasons, causes a greater number of hospital admissions due to respiratory infection than the influenza or parainfluenza viruses.

\section{ACKNOWLEDGEMENTS}

This study was funded in part by grant no. 200411079 from the Department of Health of the Basque Government.

\section{DECLARATION OF INTEREST}

None.

\section{REFERENCES}

1. van den Hoogen BG, et al. A newly discovered human pneumovirus isolated from young children with respiratory tract disease. Nature Medicine 2001; 7: 719-724.

2. Kahn JS. Epidemiology of human metapneumovirus. Clinical Microbiology Reviews 2006; 19: 546-557.

3. Sloots TP, et al. Human metapneumovirus, Australia, 2001-2004. Emerging Infectious Diseases 2006; 12 $1263-1266$.

4. Williams JV, et al. Human metapneumovirus and lower respiratory tract disease in otherwise healthy infants and children. New England Journal of Medicine 2004; 350: 1788-1790.

5. van den Hoogen BG, et al. Prevalence and clinical symptoms of human metapneumovirus infection in hospitalized patients. Journal of Infectious Diseases 2003; 188: 1571-1577.

6. Peiris JS, et al. Children with respiratory disease associated with metapneumovirus in Hong Kong. Emerging Infectious Diseases 2003; 9: 628-633.

7. Madhi SA, et al. Seasonality, incidence, and repeat human metapneumovirus lower respiratory tract infections in an area with a high prevalence of human immunodeficiency virus type-1 infection. Pediatric Infectious Disease Journal 2007; 26: 693-699.

8. Weigl JAI, et al. Ten years' experience with year-round active surveillance of up to 19 respiratory pathogens in children. European Journal of Pediatrics 2007; 166: 957-966.

9. Gerna G, et al. Changing circulation rate of human metapneumovirus strains and types among hospitalized pediatric patients during three consecutive winter-spring seasons. Archives of Virology 2005; 150: 2365-2375.

10. Williams JV, et al. The role of human metapneumovirus in upper respiratory tract infections in children: a 20-year experience. Journal of Infectious Diseases 2006; 193: $387-395$

11. Coirás MT, et al. Simultaneous detection of fourteen respiratory viruses in clinical specimens by two multiplex reverse transcription nested-PCR assays. Journal of Medical Virolology 2004; 72: 484-495.

12. Fouchier RA, et al. Detection of influenza A viruses from different species by PCR amplification of conserved sequences in the matrix gene. Journal of Clinical Microbiology 2000; 38: 4096-4101.

13. Steininger C, Aberle SW, Popow-Kraupp T. Early detection of acute rhinovirus infections by a rapid reverse transcription-PCR assay. Journal of Clinical Microbiology 2001; 39: 129-133.

14. Drosten $\mathbf{C}$, et al. Identification of a novel coronavirus in patients with severe acute respiratory syndrome. New England Journal of Medicine 2003 ; 348 : 1967-1976.

15. Vabret A, et al. Human coronavirus NL63, France. Emerging Infectious Diseases 2005; 11: 1225-1229.

16. Xu W, McDonough MC, Erdman DD. Species-Specific identification of human adenoviruses by a multiplex PCR assay. Journal of Clinical Microbiology 2000; 38 : 4114-4120.

17. Kaida A, et al. Seasonal distribution and phylogenetic analysis of human metapneumovirus among children in Osaka City, Japan. Journal of Clinical Virology 2006; 35: 394-399.

18. Wolf DG, et al. Comparison of human metapneumovirus, respiratory syncytial virus and influenza A virus lower respiratory tract infections in hospitalized young children. Pediatric Infectious Diseases Journal 2006; 25: 320-324.

19. Foulongne $\mathbf{V}$, et al. Human Metapneumovirus infection in young children hospitalized with respiratory tract disease. Pediatric Infectious Diseases Journal 2006; 25 : 354-359.

20. Garcia-Garcia ML, et al. Prevalence and clinical characteristics of human metapneumovirus infections in hospitalized infants in Spain. Pediatric Pulmonology $2006 ; 41: 863-871$.

21. Wilkesmann A, et al. Human metapneumovirus infections cause similar symptoms and clinical severity as respiratory syncytial virus infections. European Journal of Pediatrics 2006; 165: 467-475.

22. Heikkinen T, et al. Human metapneumovirus infections in children. Emerging Infectious Diseases 2008; 14: 101106. 
23. Vicente D, et al. Hospitalization for respiratory syncytial virus in the paediatric population in Spain. Epidemiology and Infection 2003; 131: 867-872.

24. Montes M, et al. Influenza-related hospitalisations among children aged less than 5 years old in the Basque Country, Spain: a 3-year study (July 2001-June 2004). Vaccine 2005; 23 : 4302-4306.
25. Mullins JA, et al. Human metapneumovirus infection among children hospitalized with acute respiratory illness. Emerging Infectious Diseases 2004; 10: 700-705.

26. von Linstow ML, et al. Excretion patterns of human metapneumovirus and respiratory syncytial virus among young children. European Journal of Medical Research 2006; 11 : 329-335. 